\title{
la tomographie sismique comme méthode de reconnaissance détaillée du sous-sol
}

\author{
exemple d'application au contrôle des injections
}

\author{
PH. COTE R. LAGABRIELLE
}

Laboratoire Central des Ponts et Chaussées".

\section{Résumé}

Après des rappels sur les tomographies en général, cet article décrit comment la tomographie sismique peut être utilisée à l'échelle du génie civil pour obtenir trois images différentes et complémentaires du sous-sol : tomographie en vitesse de propagation des ondes mécaniques, tomographie en facteur de qualité, tomographie en variation relative de la vitesse. A titre d'exemple, le contrôle de l'efficacité d'une injection illustre une application de cette technique.

\section{Abstract}

First we describe what is generally understood under the word tomography. Then the paper shows how seismic tomography can be applied to civil engineering in order to obtain three different - and complementary - images of the soil : seismic wave velocity tomography, $Q$-factor tomography and relative velocity variation tomography. As an example, the control of grouting efficiency illustrates this technique. 
Les techniques d'exploration du sous-sol par transparence sismique entre forages jouissent d'une certaine renommée. Elles sont par exemple systématiquement utilisées lorsque l'on étudie les fondations d'une centrale nucléaire et que le comportement du sol au moment d'un séisme doit être prévu. On donne à ces techniques le nom de "cross hole ". La méthode de mise en cuvre la plus simple consiste à placer un émetteur d'ébranlement mécanique à une profondeur connue dans un forage et à mesurer, au moyen de géophones placés dans deux autres forages alignés avec le forage source et à la même profondeur que l'émetteur, les temps de propagation des ondes de compression et de cisaillement entre émetteur et récepteur. En faisant varier la profondeur, on détermine ainsi la variation entre les forages des caractéristiques élastiques moyennes du sous-sol. On peut aussi utiliser seulement deux forages coplanaires et, pour une position de l'émetteur dans un des forages, mesurer les temps de propagation des ondes tout au long du forage récepteur, puis faire varier la profondeur de l'émetteur. On obtient ainsi une collection de temps qui correspondent à des trajets d'ondes mécaniques s'entrecroisant de manière plus ou moins dense dans le terrain situé entre les deux forages. On peut alors résoudre le problème inverse et calculer une répartition de la vitesse des ondes mécaniques dans le plan des forages (fig. 1 : tomographie en vitesse). Si on mesure aussi les amplitudes des signaux reçus, on peut calculer une répartition d'une grandeur caractéristique de l'atténuation intrinsèque dans le milieu, par exemple le facteur de qualité $\mathrm{Q}$ dont nous parlerons plus en détail plus loin, on obtient ainsi une tomographie en facteur de qualité.

Après un aperçu sur les techniques de tomographie en général, nous montrerons ce qui distingue la tomographie sismique du sous-sol des autres types de tomographie, puis nous parlerons du phénomène d'atténuation des ondes mécaniques dans le sous-sol en discutant plus particulièrement de la notion de facteur de qualité et du modèle de comportement des matériaux qu'elle sousentend.

Nous expliquerons comment les tomographies en vitesse et en facteur de qualité sont obtenues et nous introduirons plus en détail une autre tomographie : la tomographie en variation relative des vitesses dont l'application principale, développée jusqu'à présent, est le contrôle des injections. Elle est obtenue à partir de l'interprétation des différences de temps de trajet des ondes mesurés avant et après l'injection de coulis dans le sol.

a)

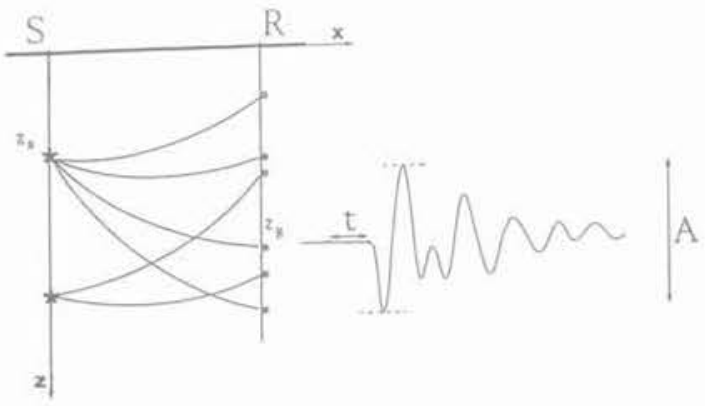

SUR FACE

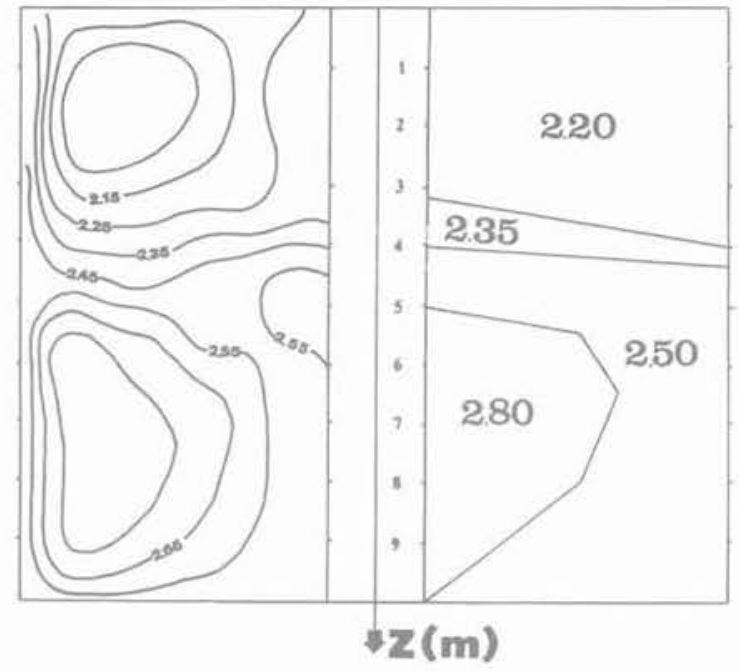

Fig. 1 - Principe de la tomographie acoustique entre forages.

a) dispositif de mesure :

$Z_{s}$ : profondeurs successives de la source.

$Z_{R}$ : profondeurs successives des récepteurs.

$t$ : temps de trajet mesuré sur un signal typique.

A : amplitude des deux premières alternances.

b) résultat d'une tomographie (vitesses) calculée à partir de mesures artificielles sur le modèle de droite.

\section{LES TECHNIQUES DE TOMOGRAPHIE}

Le mot tomographie est composé de deux racines grecques et signifie description ou dessin d'une coupe. Il y a donc là deux idées. La première est la réalisation d'une coupe de l'objet que l'on désire explorer. Cette coupe est naturellement non destructive. La deuxième est d'en représenter une image.

Il y a plusieurs manières de classer les tomographies. La première consiste à considérer le type de phénomène physique qui est utilisé. On distingue aussi les tomographies d'émission des tomographies de transmission. Certaines images sont obtenues de manière analogique et d'autres par le calcul. On peut enfin considérer le domaine d'application.

Les tomographies de transmission utilisent les propriétés d'interaction entre une onde et la matière à travers laquelle elle se propage. L'onde est générée par une source extérieure ; on mesure le signal qu'elle produit sur un ou plusieurs capteurs disposés de l'autre côté de l'objet. Les données du problème inverse à partir desquelles on construira limage sont constituées de l'ensemble de toutes les mesures qui résultent de l'interaction de l'onde avec l'objet dans toutes les directions du plan de coupe (fig. 2). Les tomographies de transmission utilisent les ondes électromagnétiques dans un large spectre de fréquences : des radiofréquences aux rayons $\gamma$ en passant par les micro-ondes et les rayons X. La technique la plus connue est celle que l'on appelle couramment * Scanner ». Il s'agit d'une tomographie aux rayons $\mathrm{X}$ assistée par ordinateur; c'est une technique de diagnostic médical. La tomographie sismique est aussi une tomographie de transmission. 


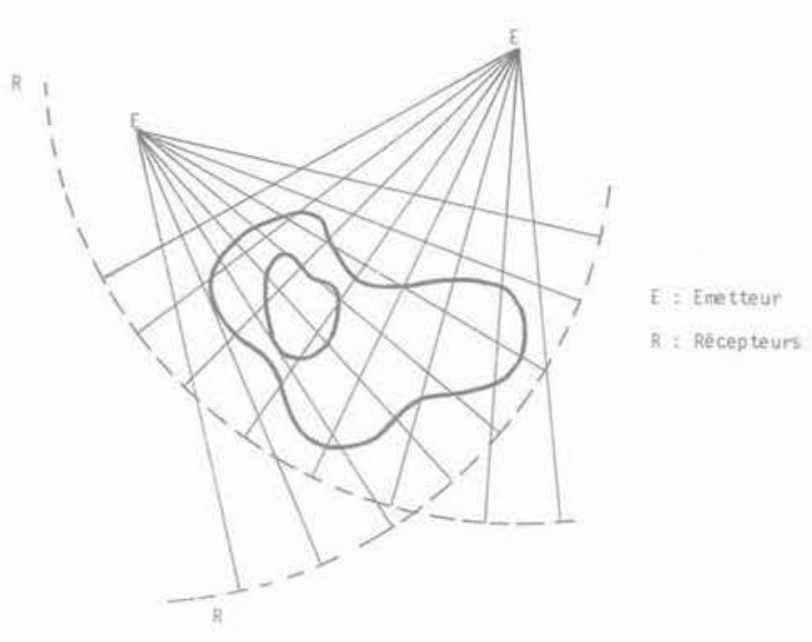

Fig. 2 - Schéma de principe d'une tomographie de transmission.

L'ensemble du dispositif émetteur-récepteurs tourne autour de l'objet à explorer.

Les tomographies d'émission utilisent un principe différent : la matière est elle-même productrice d'un phénomène physique dont on mesure les effets de l'extérieur. L'opération d'interprétation consiste à rechercher la répartition des * sources " (radio-actives par exemple) à l'intérieur de l'objet. De même on peut envisager de rechercher la répartition d'un corps feromagnétique à l'intérieur d'un objet en observant à l'extérieur le champ magnétique qu'il produit.

Les tomographies de transmission du domaine médical présentent trois caractéristiques qui les distinguent de manière importante de la tomographie acoustique utilisée pour explorer le sous-sol :

1. Les ondes utilisées sont monochromatiques.

2. L'objet à explorer - le corps humain - est limité dans toutes les directions, ce qui permet de l'éclairer dans toutes les directions.

3. Les contrastes de vitesse des ondes à l'intérieur des tissus sont très faibles. On est donc en général autorisé à considérer que les ondes se propagent suivant des rais rectilignes.

Dans ces conditions, on démontre que les mesures correspondant à une position de la source peuvent être formulées en termes d'une restriction de la transformée de Fourier à deux dimensions de la fonction qui décrit la répartition de la propriété intéressante de la matière dans l'objet (la masse volumique dans le cas où l'on utilise des rayons X). En faisant varier la position de la source et des récepteurs on peut alors reconstituer cette transformée de Fourier. On obtient la tomographie en calculant la transformée de Fourier inverse. Cette opération s'appelle rétroprojection. (Pour cela l'hypothèse $n^{\circ} 3$ ci-dessus n'a pas absolument besoin d'être vérifiée car la formulation du problème en termes de transformées de Fourier peut toujours être faite si l'on tient compte des diffractions des ondes à l'intérieur de lobjet). (HERMAN, 1980).

Revenons à la tomographie sismique dans le sous-sol. Les trois caractéristiques ci-dessus sont modifiées :

1. Les ébranlements mécaniques émis sont de nature impulsionnelle, donc à spectre de fréquence étalé.
2. Le sous-sol n'est limité que vers le haut. Pour l'explorer on utilise des forages et l'éclairement est incomplet : les techniques d'inversion des mesures par rétroprojection sont donc délicates à utiliser.

3. Les contrastes de vitesse des ondes mécaniques sont souvent forts dans le sous-sol : les rais sismiques sont exceptionnellement rectilignes. Cela signifie que lorsque l'on mesure un temps de trajet entre un émetteur et un récepteur, on ne connaît pas la géométrie du trajet.

\section{TOMOGRAPHIE EN V ET TOMOGRAPHIE EN Q}

Les grandeurs caractéristiques du terrain dont on veut retrouver la répartition entre les forages sont la vitesse des ondes mécaniques $V_{p}$ (ondes de compression) ou $V_{s}$ (ondes de cisaillement), dont les définitions sont bien connues et très claires, ainsi que le facteur de qualité $\mathrm{Q}$, dont les définitions sont nombreuses et plus ou moins équivalentes mais moins bien connues. La répartition des vitesses est calculée à partir de l'ensemble des résultats de mesure des temps de trajet. La répartition de $Q$ est calculée à partir de l'ensemble des résultats de mesure d'amplitudes.

\section{Généralités sur la notion de facteur de qualité}

Un article de BOURBIE (1985) fait un large tour d'horizon sur les connaissances actuelles en matière de mécanismes d'atténuation de l'amplitude des ondes sismiques ou acoustiques. Il passe en revue les diverses définitions de $\mathrm{Q}$ et nous ne les discuterons pas ici. Nous nous contentons d'introduire le sujet, d'illustrer les différents phénomènes d'atténuation et d'indiquer quel mode de comportement de la matière est sous-entendu lorsque l'on modélise l'atténuation intrinsèque en utilisant la notion de facteur de qualité.

L'expérience montre que l'amplitude d'une onde plane monochromatique décroît exponentiellement avec la distance parcourue par le front d'onde et peut être écrite sous la forme:

$\mathrm{X}$ : distance parcourue

$$
A(x)=A_{0} \exp \{-\alpha x\}
$$

$A_{0}$ : amplitude à lorigine.

On remarque que $\alpha$ est grossièrement proportionnel à la fréquence et ne dépend pas de $\mathrm{A}_{0}$ tant que les déformations sont inférieures à $10^{-6}$. (KJARTANSSON, 1979). Ceci suggère fortement que la réponse en amplitude des matériaux est dominée par des effets linéaires. Pour tenir compte de ces observations on définit un paramètre, nommé facteur de qualité, indépendant de la fréquence (tout au moins sur une bande limitée) en posant :

$$
\alpha=\frac{\pi \mathrm{f}}{\mathrm{Q} . \mathrm{V}}
$$

$\mathrm{f}$ : fréquence

$\mathrm{V}$ : vitesse de l'onde considérée

$\mathrm{Q}$ : facteur de qualité (sans dimension)

Pour respecter le principe de causalité, l'utilisation d'un $\mathrm{Q}$ constant impose que la vitesse de l'onde dépende de 
la fréquence. Comme l'expérience n'a pas permis jusqu'à présent de mettre clairement en évidence ce phénomène de dispersion, on choisit une loi de variation de la vitesse en fonction de la fréquence qui rende la dispersion minimale tout en assurant la causalité (fig. 3).

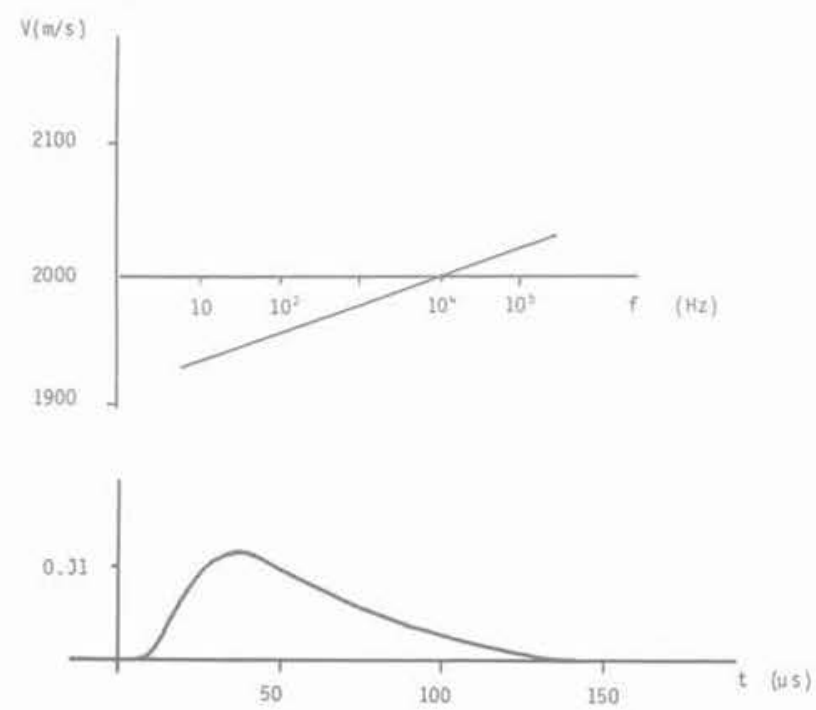

Fig. 3 - En haut : Dispersion nécessaire à la vitesse de l'onde en fonction de la fréquence, dans un milieu atténuant à $Q$ constant, afin d'assurer la causalité.

En bas: Résultat de la propagation causale d'une impulsion (Dirac à la source)

dans un milieu atténuant à $Q$ constant.

L'origine des temps est translatée du temps de trajet à la vitesse de référence.

Les formules ci-dessus montrent que les fortes valeurs de $\mathrm{Q}$ correspondent à des matériaux qui transmettent bien l'énergie élastique tandis que les faibles valeurs de $\mathrm{Q}$ correspondent à des matériaux atténuants. Dans la pratique, on constate que des matériaux sans cohésion présentant une fissuration ouverte ont des valeurs de $Q$ souvent inférieures à 20 tandis que des matériaux très cohérents et non fissurés ou à fissures fermées peuvent avoir des valeurs de Q supérieures à 100 .

La détermination de la répartition de $Q$ peut donc permettre d'atteindre la répartition de la fissuration ouverte dans le sous-sol. Q est d'ailleurs beaucoup plus sensible à la fissuration que la vitesse des ondes de compression (voir par exemple: NEWMAN et WORTHINGTON, 1982).

Lorsque l'on a affaire à une onde mécanique plane dans un milieu homogène, l'atténuation de l'amplitude ne dépend que des phénomènes anélastiques dont le matériau est le siège et la notion de facteur de qualité sert à quantifier globalement ces phénomènes.

En dehors de latténuation anélastique, deux mécanismes doivent aussi être pris en compte pour expliquer latténuation de l'amplitude :

- Le premier est l'atténuation géométrique. L'amplitude diminue avec l'augmentation de surface des fronts d'onde lorsqu'ils s'éloignent de la source située dans un domaine limité. Ainsi pour une source à symétrie sphérique, dans un milieu élastique homogène, l'amplitude des ondes de volume décroît comme $1 / \mathrm{R}$ si $\mathrm{R}$ est la distance au centre de la source. Dans le cas général où les rais sont courbes, on peut calculer l'atténuation géométrique en écrivant que l'énergie élastique se conserve dans un tube de rais (JOBERT, 1973). Il faut donc absolument connaître la géométrie des rais (donc la répartition des vitesses) pour la calculer.

- Le deuxième mécanisme est celui de la réflexion ou de la réfraction des fronts d'ondes (et des rais) aux interfaces entre milieux d'impédances acoustiques différentes. Cet effet, faible en général, par rapport aux deux précédents, varie avec l'incidence de l'onde sur l'interface. Pour des incidences voisines de la normale, et des contrastes d'impédance peu élevés, on peut le négliger (fig. 4).

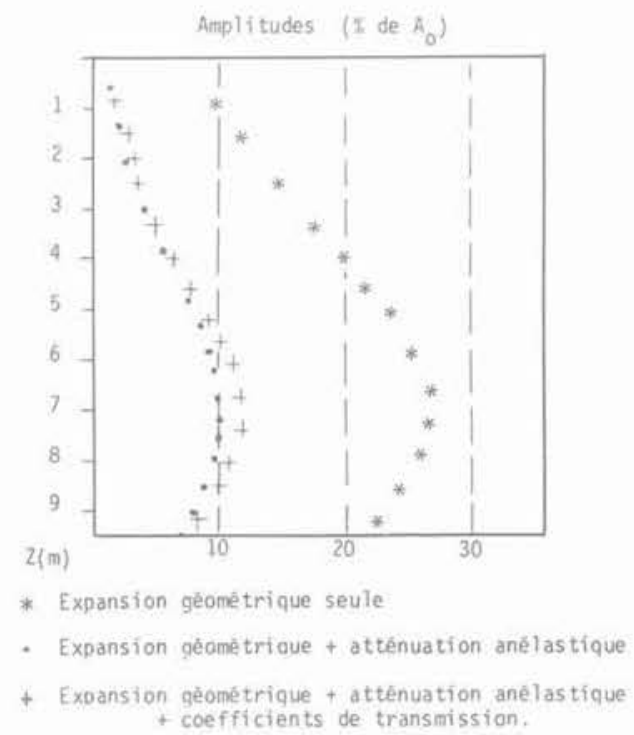

Fig. 4 - Influences relatives des différentes causes de l'atténuation de l'amplitude des déplacements pour une onde $P$ se propageant dans un terrain constitué de

deux zones à vitesses différentes et de même $Q$, séparées par une interface verticale.

La source est située à $7 m$ de profondeur.

Les récepteurs sont situés dans un forage distant de $4 m$ du forage source.

Les trois courbes représentent respectivement : l'amplitude d'un signal calculé en ne tenant compte que de l'expansion géométrique; idem en tenant compte de l'expansion géométrique et de l'atténuation anélastique; idem avec expansion géométrique, atténuation anélastique et coefficients de transmission à l'interface.

Les données nécessaires à la détermination de $Q$ peuvent être constituées par l'amplitude crête-crête des deux premières alternances du signal (fig. 1). Ceci conduit à des résultats interprétables mais dont la qualité est moins bonne que celle obtenue pour des tomographies en vitesse. Cela a plusieurs causes :

- Premièrement, l'amplitude est toujours plus difficile à mesurer que le temps de trajet car les sources sismiques sont rarement parfaitement stables en puissance (elles le sont plus facilement en contenu fréquentiel) ; - Deuxièmement, l'amplitude dépend non seulement des phénomènes anélastiques, mais aussi de l'expansion géométrique des fronts d'ondes et des pertes d'éner- 
gie dues à la présence d'hétérogénéités dans le milieu. Ces effets ne sont pas toujours faciles à corriger.

Pour ces raisons, il s'avère intéressant de chercher dans le début du signal une autre grandeur qui ne soit fonction que de l'atténuation intrinsèque. Le temps de montée $-\tau$ - (STRICK, 1970) répond bien à ces impératifs (fig. 5). C'est une mesure de temps, elle ne dépend donc pas des variations en puissance de la source; d'autre part, l'expérience, relayée par la théorie, (GLADWIN and STACEY, 1974 : BLAIR and SPATHIS, 1982) montre que $\tau$ est relié linéairement à $1 / \mathrm{Q}$ :

$$
\tau=\tau_{0}+\mathrm{C} \int_{\text {rai }} \frac{\mathrm{dt}}{\mathrm{Q}}
$$

- C * est une constante et dt est le temps de trajet infinitésimal le long du rai. $\tau_{0}$ est le temps de montée à la source. Des mesures de $\tau$ pourront donc servir de données pour le calcul des tomographies en $\mathrm{Q}$ qui seront obtenues cette fois-ci avec une qualité analogue à celle des inversions en vitesse.
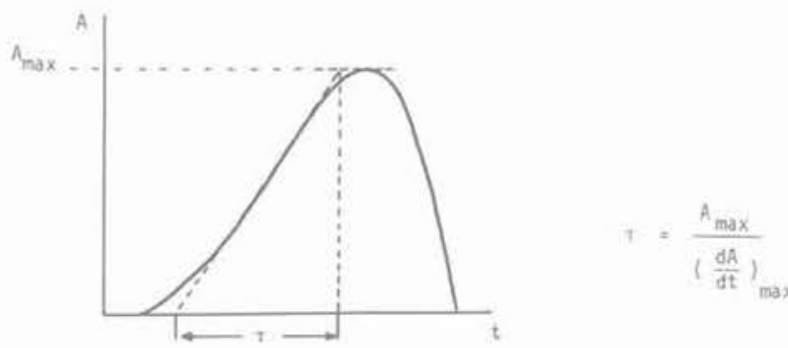

Fig. 5 - Définition possible de $\tau$ (Gladwin and Stacey, 1974).

Cette grandeur qui est reliée linéairement à l'atténuation, est indépendante des variations en puissance de la source.

\section{INVERSION DES MESURES DE TERRAIN}

Deux forages supposés coplanaires sont utilisés. Le premier reçoit une source sismique (étinceleur), le second est équipé de récepteurs (hydrophones). Pour chaque couple de positions source-récepteur, on enregistre un signal sur lequel est mesuré le temps de trajet de l'onde de compression $\left(t_{p}\right)$ ainsi que l'amplitude crête-crête de la première arrivée $\left(A_{p}\right)$ (fig. 1$)$. Le terrain est ainsi quadrillé par un grand nombre de rais sismiques. Cette opération permet de collecter les ensembles de données nécessaires aux programmes d'inversion $\left(\left\{t_{p}\right\}\right.$ et $\left.\left\{A_{p}\right\}\right)$.

Des programmes d'imagerie sismique de physique du Globe ont été adaptés à l'échelle du génie civil pour le calcul des tomographies en vitesse. Ces logiciels utilisent comme procédé d'inversion la méthode des « moindres carrés pondérés » : un milieu initial préalablement défini permet de calculer des données artificielles $\left\{t_{p a}\right\}$ qui sont comparées aux données réelles $\left\{\mathrm{t}_{p}\right\}$. Le milieu initial est alors modifié de façon à faire coïncider au mieux $\left\{\mathrm{t}_{p}\right\}$ et $\left\{\mathrm{t}_{p a}\right\}$, cette modification étant pondérée par la confiance que l'on accorde à la validité du milieu ini- tial (qui contient toutes les informations a priori : géologie, renseignements divers...).

Comme beaucoup de problèmes inverses, cette mé. thode ne conduit pas à une solution unique. Des critères de contrôle de la solution ont donc été mis au point. Ils permettent de quantifier la confiance que l'on peut accorder à telle ou telle solution, ou à telle partie de la solution (variations spatiales des critères de contrôle), Des algorithmes de même type ont pu être adaptés au facteur de qualité, ce qui permet de traiter $V p$ et $Q$ de la même façon (COTE, 1984).

\section{Application au contrôle des injections : tomographies en variations relatives des vitesses.}

Les moyens de contrôle traditionnels de l'efficacité de traitement des sols par injection de ciment ou de silicates ne sont pas satisfaisants. Il sont soit trop intégrants soit trop ponctuels. Nous avons montré que la tomographie sismique entre forages permettait de décrire les effets du traitement avec une résolution spatiale de l'ordre du mètre carré (COTE, 1984). Pour ce faire, une tomographie avant, ainsi qu'une tomographie après l'injection étaient calculées. La première décrit l'état initial du terrain et met en évidence des hétérogénéités préexistantes, tandis que la deuxième tomographie décrit l'état du terrain modifié par l'injection. L'effet en est donc apprécié par la comparaison des deux images. Il n'est pas possible de tirer des renseignements quantitatifs de cette comparaison. La simple soustraction des deux répartitions des vitesses conduirait à des résultats sans signification car chacune des deux images n'est qu'une représentation particulière dans l'espace des solutions. Si on parvient pourtant à tirer des conclusions concernant l'effet de l'injection en comparant les deux images " à l'œeil ", c'est parce que l'ensemble des solutions de chaque problème inverse est homogène et que deux représentations possibles ont suffisamment de caractéristiques communes.

Si l'on désire malgré tout obtenir une seule image ne décrivant que l'effet de l'injection, on est alors amené à effectuer une inversion unique, les données étant constituées par l'ensemble des différences entre les temps de trajets avant et après l'injection pour les mêmes positions des couples sources-récepteurs. Le résultat est une tomographie en variations relatives des vitesses.

La surface à explorer est divisée en un nombre fini de blocs rectangulaires d'indice i. Chaque rai (d'indice j) traverse un certain nombre de ces blocs dans chacun desquels la lenteur (inverse de la vitesse) est constante et égale à $s_{i}$. La longueur du trajet dans le bloc i du rai $\mathrm{d}^{\prime}$ indice $\mathrm{j}$ est $\mathrm{x}_{i j}$.

Avant linjection, la durée de propagation pour un rai donné peut s'écrire :

$$
t_{j}^{a v}=\sum_{i} \times \frac{a v}{i j} \cdot s_{i}^{a v}
$$

Après l'injection, on a de même :

$$
t_{j}^{\text {ap }}=\sum_{i} x_{i j}^{\text {ap }} \cdot s_{i}^{\text {ap }}
$$


Les données du problème inverse sont donc :

$$
\Delta t_{j}=t_{j}^{a p}-t_{j}^{a v}=\sum_{i}\left(x_{i j}^{a p} \cdot s_{i}^{a p}-x_{i j}^{a v} \cdot s_{i}^{a v}\right)
$$

On vérifie que les $\Delta t_{j}$ sont faibles. On peut donc effectuer lapproximation suivante:

$$
\mathrm{x}_{\mathrm{ij}}^{\mathrm{ap}}=\mathrm{x}_{\mathrm{ij}}^{\mathrm{av}}=\mathrm{x}_{\mathrm{ij}}
$$

On obtient :

$$
\begin{aligned}
\Delta t_{j} & =\Sigma x_{i j} \Delta s_{i} \\
\left(\Delta s_{i}\right. & \left.=s_{i}^{a p}-s_{i}^{a v}\right)
\end{aligned}
$$

Soit encore :

$$
\Delta \mathrm{T}=\mathrm{X} \Delta \mathrm{S} \text {. }
$$

Les inconnues du problème sont les variations de lenteur dans chacun des blocs, les données sont constituées des différences entre les temps de trajet mesurés après et avant l'injection. Dans la notation matricielle ci-dessus, $\Delta T$ est le vecteur colonne de $\mathrm{N}$ composantes $\Delta \mathrm{t}_{j}(\mathrm{~N}$ : nombre de rais), $\Delta \mathrm{S}$ est le vecteur colonne à $\mathrm{P}$ composantes $\Delta \mathrm{s}_{i}(\mathrm{P}$ : nombre de blocs) et $\mathrm{X}$ est la matrice $\mathrm{N} \times \mathrm{P}$ dont les éléments sont les longueurs des trajets $\mathrm{x}_{i j}$ du rai d'indice $\mathrm{j}$ dans le bloc $\mathrm{i}$. En général $\mathrm{N} \neq \mathrm{P}$ et même dans le cas où l'on choisirait $\mathrm{N}=\mathrm{P}$, le système obtenu est toujours mal conditionné. C'est pourquoi on utilise une méthode aux moindres carrés pondérés pour déterminer des estimations $\Delta \mathrm{s}$ de $\Delta \mathrm{S}$.

$\Delta T$ n'est pas de moyenne nulle, en général $\Delta S$ non plus. On est donc amené à chercher les écarts de $\Delta S$ autour d'une moyenne $\Delta \mathrm{S}_{0}$ correspondant par exemple à une variation de lenteur uniforme dans l'espace. $\Delta S_{o}$ est alors un vecteur dont toutes les composantes sont égales à :

$$
\Delta s_{o}=\frac{1}{N} \sum_{j} \frac{\Delta t_{j}}{l_{j}}
$$

$I_{\mathrm{j}}$ : longueur du rai d'indice $\mathrm{j}$.

On minimise alors la quantité :

$$
\|\mathrm{X} \Delta \mathrm{S}-\Delta \mathrm{T}\|+\theta^{2}\left\|\Delta \mathrm{S}-\Delta \mathrm{S}_{0}\right\|
$$

ce qui conduit à :

$$
\Delta S=\left(X X+\theta^{2} l\right)^{-1}\left(X \Delta T+\theta^{2} \Delta S_{o}\right)
$$

Le coefficient $\Theta^{2}$ est une mesure de la confiance relative que l'on accorde à $\Delta \mathrm{S}_{0}$. Si l'on choisit un $\Theta^{2}$ grand, cela signifie que l'on pense que l'injection a eu des effets uniformes dans l'espace. On pourrait d'ailleurs choisir un $\Delta \mathrm{S}_{o}$ dont les composantes varieraient spatialement en fonction des connaissances que l'on a par ailleurs sur les variations spatiales de l'effet de l'injection. La formu-
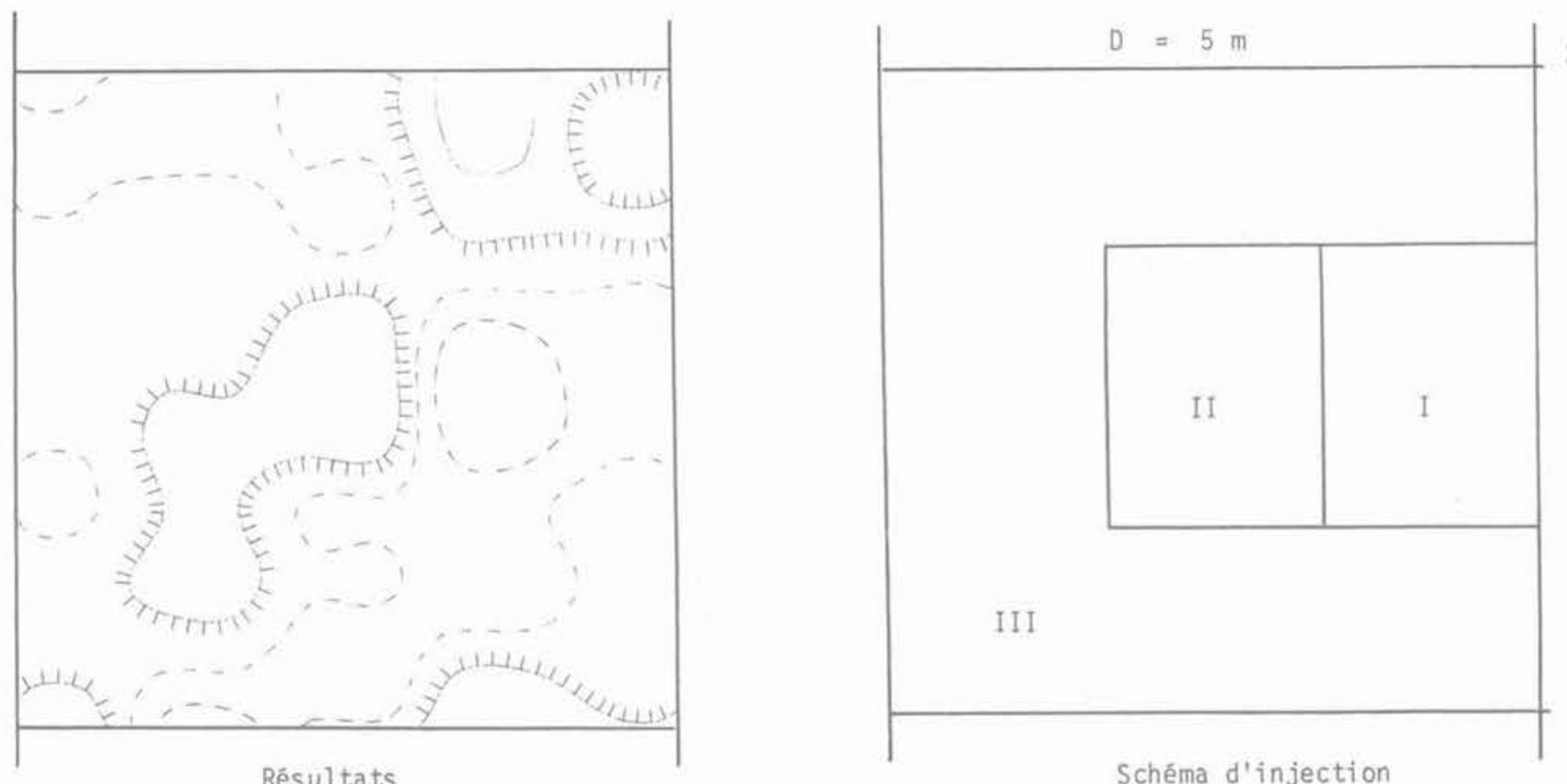

Fig. 6 - Tomographie en variations relatives de vitesse (avant - après injection).

Les courbes de niveaux sont distantes de $10 \%$. La courbe en traits pleins correspond

à une variation de $+10 \%$; la courbe hachurée à une variation nulle; les courbes en tirets correspondent à des variations négatives de la vitesse. Le terrain est subdivisé en trois zones d'injection :

Zone I: zone d'injection dense;

Zone II : zone d'injection modérée;

Zone III : pas d'injection.

On remarque que dans les zones théoriquement fortement injectées, la vitesse a fortement diminué alors qu'elle a légèrement augmenté dans leur voisinage.

Ailleurs les variations relatives de vitesse sont faibles.

On interprète ce phénomène comme étant dâ à un claquage, dans la zone l, du terrain trop imperméable vis-à-vis du coulis utilisé. Au voisinage de cette zone, le terrain a été légèrement compacté. 
lation en termes de lenteur a été utilisée, mais les résultats finaux peuvent être exprimés en termes de variations relatives de vitesse.

La figure 6 est un exemple de résultat obtenu à partir de mesures réelles dans des sables de Beauchamp sur un site d'essai d'injection à Neuilly-Plaisance (banlieue Est parisienne).

Cette méthode présente plusieurs avantages :

1. Elle fournit les résultats sous la forme d'un seul document qui ne décrit que l'effet du traitement.

2. Le système à résoudre est directement linéaire puisqu'on travaille sur des données petites (différences de temps de trajets).

3. En sismique, il est bien connu que le pointé relatif des temps est plus précis que le pointé absolu ; or cette méthode utilise principalement des pointés relatifs. Il est possible d'enregistrer les signaux avant et après l'injection, puis de calculer les différences de temps de trajet par des méthodes automatiques de traitement du signal. Ceci, en éliminant les pointés * manuels », confère une grande précision à la méthode.

4. L'effet de certaines erreurs systématiques est éliminé, par exemple celui dû à une connaisance imparfaite de la géométrie des forages.

\section{CONCLUSION}

Des simulations numériques ainsi que des expérimentations sur le terrain ont montré l'efficacité de la tomographie sismique. Il est possible de porter un jugement sur l'effet d'une injection dans le sol avec une résolution spatiale de l'ordre du mètre carré.

La tomographie en variation relative de vitesse est particulièrement bien adaptée au contrôle des injections pour lesquelles elle constitue une aide efficace au diagnostic. Elle peut s'appliquer en fait chaque fois qu'un terrain évolue mécaniquement dans le temps et nécessite un contrôle (par exemple : traitements thermiques, évolution de zones fissurées, de nappes aquatiques...). Elle est calculée en utilisant comme données les différences entre des mesures réalisées avant et après une injection. Le coût du calcul est faible, mais pour le moment la durée de la mesure sur le terrain est encore excessive pour que la méthode soit économiquement viable (il faut environ une journée de travail pour obtenir une image). Un matériel spécifique est en cours de réalisation. Il nous permettra de réduire cette durée à environ une demi-heure pour une image (mesure + calcul) dans le cas d'une tomographie d'une centaine de mètres carrés avec dix positions d'émetteur et dix positions de récepteurs.

\section{BIBLIOGRAPHIE}

1. BLAIR D.P. and SPATHIS A.T. 1982, Attenuation of explosion generated pulses in rock masses, J. Geophys. Res,, 87, pp. 3885-3892.

2. BOURBIE T. 1985, Atténuation intrinsèque des ondes sismiques. Première partie : Définitions et mesures. Revue de l'Inst. Français du Pétrole, Vol. 39, $\mathrm{n}^{\circ} 6, \mathrm{pp} .681-705$.

3. COTE Ph. 1984, Inversion en vitesse et facteur de qualité de mesures de transparence sonique entre forages. R.R. L.P.C. $n^{\circ} 132$, L.C.P.C. Paris.

4. GLADWIN M.T. and STACEY F.D 1974, Anelastic degradation of acoustic pulses in rock. Phys. of the Earth and Planetary Interiors, 8, pp. 332-336.

5. HERMAN G.T 1980, Image reconstruction from projections ; The fundamentals of computerized tomography. Acc. Press. Inc. NEW YORK.

6. JOBERT G. 1973, Ondes en milieu élastique isotrope et hétérogène. Traité de Géophys. Int., Coulomb et Jobert, MASSON, Paris, I, pp. 149-169.

7. KJARTANSSON E. 1979, Constant Q-wave propagation and attenuation. J. Geophys. Res., 84, pp. $4737-4748$.

8. NEWMAN P.J and WORTHINGTON M.H. 1982 , In situ investigation of seismic body wave attenuation in heterogeneous media. Geophys. Prospect., 30 , pp. $377-440$.

9. STRICK E. 1970, A Pedestal effect for pulse propagation in constant $-Q$ solids. Geophysics, V. 35, pp. $387-403$. 